\title{
Glucose Exposure in Peritoneal Dialysis Is a Significant Factor Predicting Peritonitis
}

\author{
Herma Uiterwijk $^{\mathrm{a}}$ Casper F.M. Franssen ${ }^{\mathrm{b}}$ Johanna Kuipers $^{\mathrm{a}}$ Ralf Westerhuis $^{\mathrm{a}}$ \\ Ferdau L. Nautab

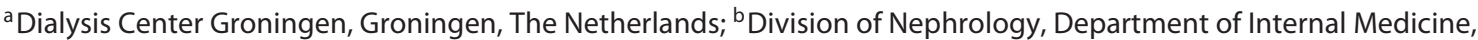 \\ University Medical Center Groningen, University of Groningen, Groningen, The Netherlands
}

\section{Keywords}

Glucose exposure · Peritoneal dialysis · Peritonitis · Residual diuresis

\begin{abstract}
Introduction: Loss of residual renal function (RRF) as well as high peritoneal glucose exposure are associated with increased peritonitis frequency in peritoneal dialysis (PD) patients. Our objective was to investigate the contribution of RRF and peritoneal glucose exposure to peritonitis in PD patients. Methods: In this prospective longitudinal cohort study, 105 incident end-stage renal disease patients that started PD between January 2006 and 2015 were studied. Follow-up was 5 years with censoring at death or switch to another treatment modality. Cox regression models were used to calculate the association between glucose exposure, RRF, and peritonitis. Kaplan-Meier analysis was used to examine the difference in occurrence of peritonitis between patients with high and low glucose exposure and between those with and without residual diuresis. Results: One hundred and five patients were followed for a mean of 23 months. Fifty-one patients developed a peritonitis. Cox regression models at 6 months showed that glucose exposure and not residual diuresis significantly predicted PD peritonitis. Kaplan-Meier analysis after 6 months of follow-up showed
\end{abstract}

that time to first PD peritonitis was significantly longer in the low glucose exposure group. Similarly, patients with RRF had a significantly longer interval to first peritonitis compared to patients without RRF. Conclusion: A higher exposure to glucose rather than loss of RRF is associated with an increased risk of peritonitis. This confirms the detrimental effects of glycemic harm to the peritoneal host defense on invading microorganisms and argues for the use of the lowest PD glucose concentrations possible.

(c) 2020 The Author(s)

Published by S. Karger AG, Basel

\section{Introduction}

Worldwide, approximately 272,000 patients are treated with peritoneal dialysis (PD) because of end-stage renal disease [1]. The most common complication of PD is peritonitis which is associated with loss of ultrafiltration, hospitalization, catheter loss, technique failure, transfer to hemodialysis (HD), and considerable mortality $[2,3]$.

The most frequent etiological agents of PD-associated peritonitis worldwide are gram-positive cocci such as Staphylococcus epidermidis and other coagulase-negative Staphylococci and Staphylococcus aureus [4].

\begin{tabular}{ll}
\hline KARGER & $\begin{array}{l}\text { Karger } \\
\text { Published by S. Karger AG, Basel The Author(s) }\end{array}$ \\
karger@karger.com & This article is licensed under the Creative Commons Attribution- \\
www.karger.com/ajn & NonCommercial-NoDerivatives 4.0 International License (CC BY- \\
NC-ND) (http://www.karger.com/Services/OpenAccessLicense). \\
Usage and distribution for commercial purposes as well as any dis- \\
tribution of modified material requires written permission.
\end{tabular}

Herma Uiterwijk

Dialysis Center Groningen

Hanzeplein 1

NL-9713 GZ Groningen (The Netherlands)

E-Mail h.uiterwijk@dcg.nl 
Previous studies have identified various modifiable (malnutrition, the use of immunosuppressive drugs, patient training) and nonmodifiable risk factors (age, gender, diabetes, residual renal function $[R R F]$ ) for peritonitis $[2,5]$. There is debate on whether high glucose exposure is a risk factor for peritonitis [6]. Long-term exposure to dialysis solutions may cause structural changes to the peritoneum and have a causative role in changes in peritoneal function. Changes in membrane function as a consequence of peritonitis are associated with recurrent peritoneal infections [6-8]. Studies that have specifically investigated the relation between high peritoneal glucose exposure and peritonitis frequency yield varying results. Some did not find a significant association between glucose exposure and peritonitis frequency $[9,10]$, whereas another study showed a significant increase in the incidence of relapsing and recurrent peritonitis in patients with a higher glucose exposure [11].

Several studies have shown the association between a decline in residual renal function and an increased risk of peritonitis [12-15]. Presently, it is unknown whether this is a direct association or whether it is mediated by other factors such as a higher glucose exposure. Patients with a decrease in RRF and loss of diuresis are almost inevitable treated with higher glucose concentrations to achieve sufficient peritoneal ultrafiltration, thus leading to a higher glucose exposure to the peritoneum [16]. Thus far, no studies investigated the relation between peritonitis frequency and RRF with taking the glucose exposure into account. Therefore, the aim of this study was to unravel the association between RRF, glucose exposure, and peritonitis rate.

\section{Patients and Methods}

\section{Patients}

In this longitudinal single-center cohort study with prospective data collection, all patients who visited our out-patient clinic between January 2006 and 2015 were eligible. All adult $(\geq 18$ years) patients that started PD therapy from the Dialysis Center Groningen were included (both continuous ambulatory PD and automatic PD). Follow-up was 5 years with censoring at death or until termination of PD treatment, whichever occurred first. Patients who had a peritonitis within 6 weeks of starting PD were excluded from the study since the possibility that the peritonitis was caused by the PD catheter insertion could not be ruled out. As measure for RRF, we primarily used the 24-h urine volume, since peritoneal glucose exposure is more closely related to volume homeostasis than to creatinine clearance $(\mathrm{CrCl})$. As a sensitivity analysis, we used the mean of the urea and $\mathrm{CrCl}$ instead of residual diuresis as measure for RRF.

\section{PD Treatment}

The PD solutions prescribed were continuous ambulatory PD/ DPCA 2 (glucose concentration 1.5\%), 3 (4.25\%), and 4 (2.3\%) from Fresenius Medical Care (Bad Homburg, Germany). Peritonitis was defined in line with the International Society for PD guidelines definition as cloudy dialysate with a dialysate white cell count of $>100$ cells $/ \mu \mathrm{L}$ and $>50 \%$ polymorphonucleaire leucocytes. $\mathrm{PD}$ peritonitis was treated according to the most recent version of the International Society for PD committee guidelines [17].

Causes of discontinuation of PD were categorized as switching to $\mathrm{HD}$, renal transplantation, or death.

\section{Data Collection}

All patients that started PD between January 2006 and 2015 were included. Follow-up was terminated at January 2016. Patient characteristics and clinical data including diuresis volumes were collected at the start of PD, at 6 weeks after the start of PD and at 6 months, 1 year and, next, annually for 5 years or until the end of PD. Patients with a urine production of $>200 \mathrm{~mL} / 24 \mathrm{~h}$ were considered to have residual diuresis. There was no loss to follow-up.

For each patient, the glucose exposure was calculated at 6 weeks after the start of PD, after 6 months, after 1 year, and next annually for 5 years or until the end of PD. The total glucose exposure in grams per $24 \mathrm{~h}$ was calculated as followed: As an example: a patient with a PD schedule of 2 dwells of $2 \mathrm{~L} 2.3 \%$ glucose and 2 dwells of 2 L 1.5\% glucose: $(2 \times 2 \times 23 \mathrm{~g})+(2 \times 2 \times 15 \mathrm{~g})=152$ g glucose

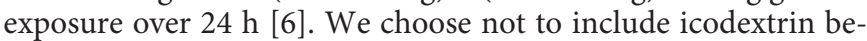
cause this water-soluble polysaccharide has very different effects on the peritoneal membrane compared to glucose. $\mathrm{CrCl}$ was calculated using the following formula: $\mathrm{CrCl}=$ (urinary creatinine $\times$ serum creatinine/24-h urine volume)/1.44.

\section{Statistical Analysis}

Statistical analysis was performed using SPSS for Windows software, version 20.0 (SPSS Inc., Chicago, IL, USA). Normally distributed data were expressed as mean \pm SD and categorical data as number (\%). Independent risk factors for peritonitis were assessed in univariate and multivariate Cox's proportional hazard models. In the multivariate model, we adjusted for sex, age, residual diuresis, daily peritoneal glucose exposure, serum albumin, and use of immunosuppressive drugs. $p$ values $<0.05$ were considered significant. For the Kaplan-Meier analyses of glucose exposure, patients were stratified in 2 groups: group 1: glucose exposure $0-120$ g/24 h and group 2: glucose exposure $>120$ g/24 h. Further Kaplan-Meier analysis was performed after residual diuresis. Therefore, patients were divided in 2 groups: group 1 had residual diuresis. Group 2 was anuric. Continuous data were used in de multivariate models. Comparisons of peritonitis frequency were performed using Kaplan-Meier method.

\section{Results}

\section{Patient Characteristics and Clinical Data}

As shown in Table 1, the mean age was $52.8 \pm 17.4$ years and $59 \%$ were male. About $87 \%$ of the patients had residual diuresis. Failure of a previous renal transplant and $\mathrm{HD}$ before PD are the main reasons of loss of residual di- 
uresis. Nineteen percent of the patients had previous kidney transplantation and $2 \%$ heart transplantation. Twenty-four percent of the patients used immunosuppressive drugs (Table 1). Mean follow-up time was $22.9 \pm$ 20.3 months. A total of $49 \%$ of the patients experienced at least one peritonitis. Patients were on dialysis for $16.9 \pm$ 18.6 months at the first episode of peritonitis. The average peritoneal glucose exposure at 6 weeks and after 6 months was $119.3 \pm 36.0$ and $141.6 \pm 48.2 \mathrm{~g} / 24 \mathrm{~h}$, respectively (Table 2). The glucose exposure during follow-up remained fairly stable (at 1 year: $145 \pm 49 \mathrm{~g} / 24 \mathrm{~h}$; at 2 years: $155 \pm 61 \mathrm{~g} / 24 \mathrm{~h}$; at 3 years: $152 \pm 40 \mathrm{~g} / 24 \mathrm{~h}$; at 4 years: $173 \pm 57 \mathrm{~g} / 24 \mathrm{~h}$; at 5 years: $152 \pm 46 \mathrm{~g} / 24 \mathrm{~h}$ ). After 6 months, $75 \%$ of the patients had residual diuresis. At time of peritonitis, $69 \%$ had residual diuresis.

\section{Residual Diuresis, Glucose Exposure, and Peritonitis Incidence}

Results of univariate Cox regression analyses are listed in Table 3. These data show that in univariate analysis, the use of immunosuppressive drugs and serum albumin (as parameter for nutritional status) significantly predicted time to first peritonitis $(p \leq 0.001$ and $p=0.001$ respectively). Residual diuresis at baseline $(p=0.50)$ or glucose exposure at baseline $(p=0.10)$ did not significantly predict peritonitis. If the analyses was performed with the mean of the urea and $\mathrm{CrCl}$ instead of residual diuresis as measure for RRF similar results were obtained.

However, Cox regression with the same parameters at 6 months yielded different results. After 6 months of follow-up, both residual diuresis and glucose exposure were significant predictors for peritonitis $(p=0.038$ and $p<$ 0.001 , respectively).

The multivariate model (Table 3 ) yielded identical results. In a multivariate model adjusting for age, sex, residual diuresis, daily glucose exposure, serum albumin, and use of immunosuppressive drugs, the use of immunosuppressive drugs and serum albumin significantly predicted peritonitis ( $p \leq 0.001$ and $p=0.004$, respectively). Furthermore, it showed that after 6 months of PD treatment, glucose exposure significantly predicted peritonitis $(p=0.024)$ whereas residual diuresis did not reach significance $(p=0.75)$.

Both univariate and multivariate analyses showed that peritoneal glucose exposure at 1 year after the start of $\mathrm{PD}$ also predicted peritonitis (hazard ratio [HR] 4.3, 95\% CI [2.3-8.2], $p \leq 0.001$ and HR 4.0, 95\% CI [1.72-9.46], $p=$ 0.001 , respectively), whereas residual diuresis was not significant. Analysis at 2 years after the start of PD showed
Table 1. Baseline patient characteristics and clinical data

\begin{tabular}{lc}
\hline Characteristic & $n=105$ \\
\hline Age, years & $52.8 \pm 17.4$ \\
Gender, male & $62(59)$ \\
Weight, kg & $75.5 \pm 13.3$ \\
Length, cm & $173 \pm 10.8$ \\
BMI, kg/m ${ }^{2}$ & $25.2 \pm 3.6$ \\
Residual diuresis & $91(87)$ \\
CrCl, mL/min & $7.5 \pm 9.9$ \\
Urea - CrCl, mL/min & $10.0 \pm 6.1$ \\
Diabetes & $10(9.5)$ \\
Hypertension & $99(94)$ \\
Cardiovascular disease & $37(35)$ \\
Previous kidney transplantation & $20(19)$ \\
Previous heart transplantation & $2(2)$ \\
Albumin, g/L & $38.6 \pm 4.8$ \\
Immunosuppressive drugs & $25(24)$ \\
\hline
\end{tabular}

Categorical variables are presented as number (percentage); continuous variables are presented as mean $\pm \mathrm{SD}$.

$\mathrm{CrCl}$, creatinine clearance.

Table 2. Data during follow-up

\begin{tabular}{lc}
\hline Parameters & $n=105$ \\
\hline Follow-up, months & $22.9 \pm 20.3$ \\
Mortality during follow-up & $34(32)$ \\
Number of patients developing peritonitis & $51(49)$ \\
Peritonitis incidence, number/year & $0.07 \pm 0.14$ \\
PD modality at 6 months & \\
$\quad$ CAPD & $66(63)$ \\
APD & $39(37)$ \\
Interval to 1st peritonitis all patients, months & $16.9 \pm 18.6$ \\
Peritonitis on & \\
$\quad$ CAPD treatment & $27(41)$ \\
APD treatment & $24(62)$ \\
Number of patients without residual diuresis at & \\
$\quad$ the time of peritonitis & $17(31)$ \\
Number of patients with residual diuresis at & $75(75)$ \\
$\quad 6$ months & \\
Peritoneal glucose exposure, g/24h & $119.3 \pm 36.0$ \\
$\quad 6$ weeks & $141.6 \pm 48.2$ \\
6 months & $7.5 \pm 9.9$ \\
CrCl at 6 weeks, mL/min & \\
Number of patients in follow-up & 55 \\
At 1 year & 28 \\
At 2 years & 11 \\
At 3 years & 4 \\
At 4 years & 7 \\
At 5 years & \\
\hline
\end{tabular}

Outcome parameters during follow-up. Categorical variables are presented as number (percentage); continuous variables are presented as mean $\pm \mathrm{SD}$.

$\mathrm{CrCl}$, creatinine clearance; $\mathrm{PD}$, peritoneal dialysis; CAPD, continuous ambulatory PD; APD, automatic PD. 
Table 3. Cox regression with univariate and multivariate analysis of baseline and 6 months characteristics and the outcome parameter peritonitis

\begin{tabular}{|c|c|c|c|c|}
\hline Parameters & $\begin{array}{l}6 \text { Weeks } \\
\text { HR }(95 \% \text { CI })\end{array}$ & $p$ value & $\begin{array}{l}6 \text { Months } \\
\text { HR (95\% CI) }\end{array}$ & $p$ value \\
\hline \multicolumn{5}{|l|}{ Univariate Cox regression analysis } \\
\hline Residual diuresis & $0.77(0.35-1.66)$ & 0.50 & $0.53(0.29-0.97)$ & 0.038 \\
\hline Glucose exposure (per $100 \mathrm{~g} / 24 \mathrm{~h}$ ) & $1.99(0.87-4.56)$ & 0.10 & $2.93(1.69-5.08)$ & $<0.001$ \\
\hline Use of immunosuppressive drugs & $3.9(2.14-7.21)$ & $<0.001$ & - & - \\
\hline Serum albumin, $g / \mathrm{L}$ & $0.91(0.85-0.96)$ & 0.001 & $0.89(0.82-0.96)$ & 0.004 \\
\hline \multicolumn{5}{|l|}{ Multivariate Cox regression analysis } \\
\hline Gender & $0.92(0.46-1.85)$ & 0.82 & $0.54(0.26-1.11)$ & 0.09 \\
\hline Age, years & $1.00(0.98-1.01)$ & 0.60 & $1.00(0.98-1.02)$ & 0.92 \\
\hline Residual diuresis & $1.06(0.37-3.05)$ & 0.91 & $0.86(0.34-2.18)$ & 0.75 \\
\hline Glucose exposure (per 100 g/L) & $1.48(0.58-3.76)$ & 0.41 & $2.19(1.11-4.32)$ & 0.02 \\
\hline Immunosuppressive drugs & $4.04(2.13-7.68)$ & $<0.001$ & $3.53(1.66-7.53)$ & 0.001 \\
\hline Albumin, g/L & $0.92(0.87-0.97)$ & 0.004 & $0.91(0.83-0.99)$ & 0.02 \\
\hline
\end{tabular}

Results of univariate and multivariate Cox regression analysis with peritonitis as outcome and listed the variables as covariate.

$\mathrm{HR}$, hazard ratio.

that glucose exposure still predicted peritonitis univariately (HR 1.7, 95\% CI [1.0-2.9], $p=0.045$ ), but not multivariately; in this multivariate analysis at 2 years after the start of $\mathrm{PD}$, only the use of immunosuppressive drugs remained significant.

Kaplan-Meier analysis of the peritonitis-free survival showed that patients with a high $(>120 \mathrm{~g} / 24 \mathrm{~h}$ ) daily glucose exposure at 6 weeks and at 6 months had a shorter time to peritonitis compared to patients that had a low daily glucose exposure ( $\leq 120 \mathrm{~g} / 24 \mathrm{~h}$; Fig. 1a, b).

Kaplan-Meier analysis of the peritonitis-free survival of the patient groups categorized by the presence or absence of residual diuresis at 6 weeks and 6 months showed no significant difference $(p=0.49)$ between patients with or without residual diuresis (Fig. 1c) at 6 weeks. However, at 6 months there was a significant difference $(p=0.033$; Fig. 1d).

\section{Discussion}

The goal of this study was to unravel the association between RRF, glucose exposure, and the time to peritonitis. The major finding in our study was that after 6 months follow-up the glucose exposure is the most important risk factor for the occurrence of peritonitis, independent of residual diuresis. This suggests that higher exposure to glucose rather than loss of RRF is associated with an increased risk of peritonitis.
In line with other studies, we confirm in this study that use of immunosuppressive drugs as wel as serum albimin are both strong significant predictors of PD peritonitis. Furthermore, the study showed that at 6 weeks both residual diuresis as well as daily peritoneal glucose exposure did not predict the occurrence of peritonitis. Sensitivity analyses with the mean of the urea and $\mathrm{CrCl}$ instead of residual diuresis as measure for RRF yielded similar results.

Several studies described the protective factor of RRF for peritonitis $[2,12,18]$. In our study, we only see after 6 months of PD that residual diuresis is a protective factor for peritonitis. When adjusting for other risk factors, it fails to demonstrate a significant role for residual diuresis. Obviously, residual diuresis and glucose exposure are closely related, because patients with limited residual diuresis usually need higher glucose exposure to ensure adequate ultrafiltration. As far as we know, this study is the first that included both residual diuresis as peritoneal glucose exposure. This study showed that it is not the residual diuresis itself, but peritoneal glucose exposure that relates to the peritonitis. Several studies have investigated the role of peritoneal glucose exposure on developing peritonitis. Some studies have found no effect $[9,10]$, whereas other found that glucose exposure was a significant factor [11]. These divergent results may be explained by differences in the categorization of glucose exposure. The studies that found no significant effect of glucose exposure on peritonitis have only investigated this by dividing patients in a high and low glucose group. This ap- 


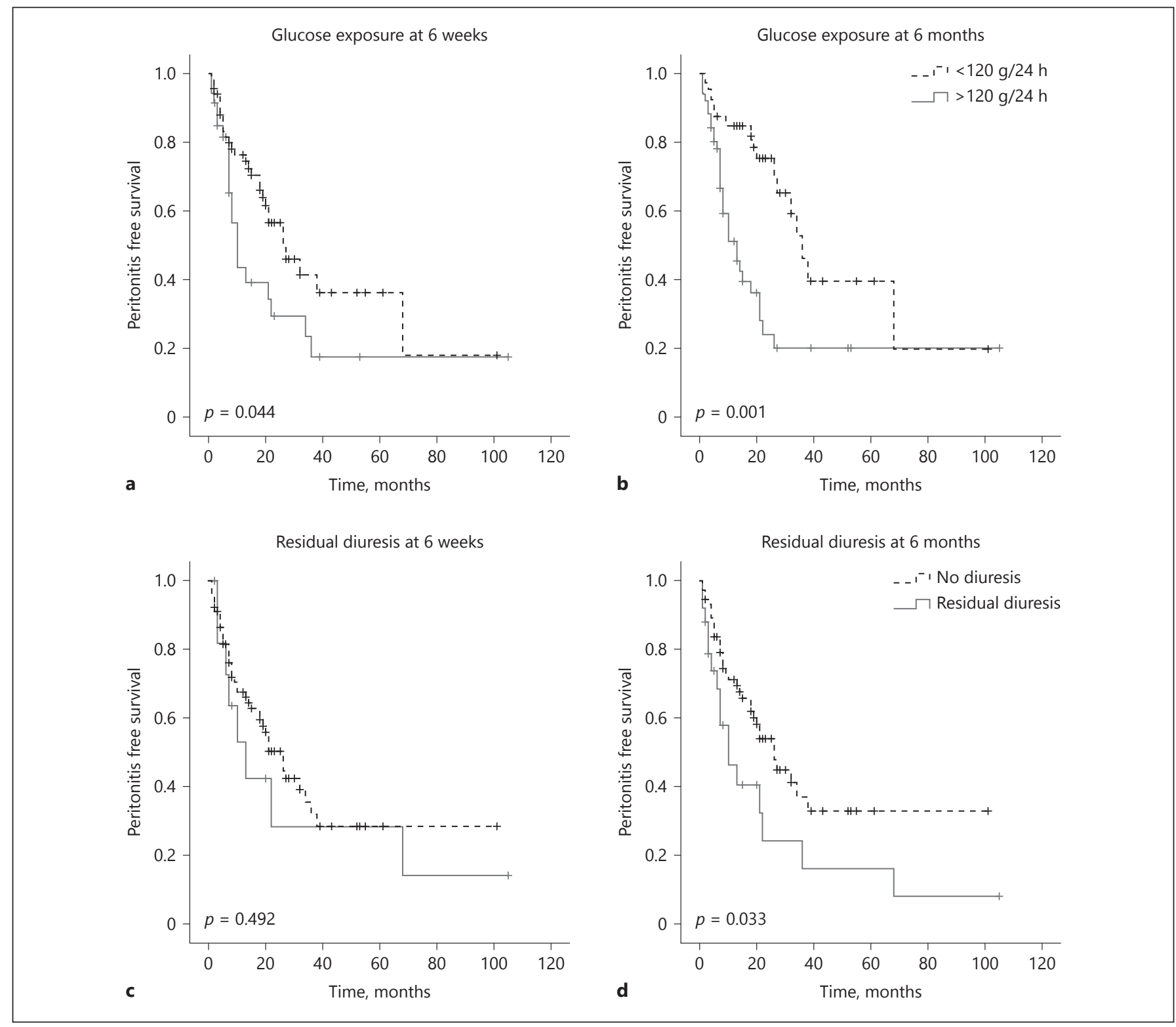

Fig. 1. a Kaplan-Meier curve showing time to first peritonitis according to glucose exposure at 6 weeks. Group 1, the dashed line, contains patients with a glucose exposure $\leq 120 \mathrm{~g} / 24 \mathrm{~h}(n=69)$; Group 2, the gray line, contains patients with a glucose exposure $>120 \mathrm{~g} / 24 \mathrm{~h}(n=35)$. b Kaplan-Meier curve showing time to first peritonitis according to glucose exposure at 6 months. Group 1, the dashed line, contains patients with a glucose exposure $\leq 120 \mathrm{~g} / 24 \mathrm{~h}$ $(n=40)$; Group 2, the gray line, contains patient with a glucose ex-

proach might not be sensitive enough to detect the effect of glucose exposure on peritonitis.

Other studies suggest that a high peritoneal glucose load increases the risk of peritonitis, perhaps as the effect of impaired host defenses, vascular disease, and damage posure $>120 \mathrm{~g} / 24 \mathrm{~h}(n=51)$. c Kaplan-Meier analysis of peritonitisfree survival of the patient groups categorized by the presence or absence of residual diuresis at 6 weeks. The gray line represents the anuric patients, the dashed line patients with residual diuresis. $\mathbf{d}$ Kaplan-Meier analysis of peritonitis-free survival of the patient groups categorized by the presence or absence of residual diuresis at 6 months. The gray line represents the anuric patients, the dashed line patients with residual diuresis. 
this explains the prevalence of peritonitis. Little is known about the glucose concentrations that are necessary to facilitate bactericidal activity in humans. In vitro studies however demonstrate that the bactericidal sugar concentrations were much higher than the maximum glucose concentrations in the dialysis solutions. In line with this, the osmolarity that was bactericidal in vitro was almost twice as high as the osmolality of a $3.86 \%$ dialysis solution $[10,22]$.

Clinical experience learns that the peritoneal membrane characteristics are fully known and developed after 6 months [23]. Therefore, we are convinced that the prescribed peritoneal glucose dose at 6 months does better reflect the true peritoneal characteristics. This is why we believe that only at 6 months the glucose exposure predicts peritonitis.

The present study has several strengths and weaknesses. For example, some potential risk factors such as socioeconomic status or personal hygiene were not included. Other relevant data representing nutritional status such as the subjective global assessment or incidence of culture negative peritonitis were also not included. Although adjustments for all major risk factors were made, residual confounding cannot be excluded due to the observational design of the study. Furthermore, this was a single center study.

Major strengths of this study were the inclusion of all incident $\mathrm{PD}$ patients visiting our center and the relative long-term follow-up time. The prospective design in that all patients were included directly from start PD and not during a random moment during their treatment we believe to be another strong plus. As already mentioned, this study is to our knowledge the first that included both residual diuresis as peritoneal glucose exposure.

In conclusion, this study showed that peritoneal glucose exposure and not residual diuresis predicts the occurrence of peritonitis. Further studies should shed more light to the mechanistical pathways that relate higher peritoneal glucose exposure to peritonitis. Future studies should further investigate which potential bactericidal properties of PD solutions might be clinically relevant. In our opinion, this demonstrates the importance of low glucose exposure in the prevention of peritonitis.

\section{Disclosure Statement}

J.K. received a general research grant not specifically related to this study. Further none declared. The results presented here have not been published previously in whole or part.

\section{Author Contributions}

H.U. included patients and collected data, designed the study, analyzed the data, and drafted the article. C.F.M.F. designed the study, interpreted the data, and revised the report. J.K. oversaw the analysis and made comments on the draft. R.W. oversaw the analysis and commented the original draft. F.L.N. compiled the data, analyzed the data, interpreted the data, and revised the draft.

\section{References}

1 Li PK, Chow KM, Van de Luijtgaarden MW, Johnson DW, Jager KJ, Mehrotra R, et al. Changes in the worldwide epidemiology of peritoneal dialysis. Nat Rev Nephrol. 2017 Feb;13(2):90-103.

2 Kerschbaum J, König P, Rudnicki M. Risk factors associated with peritoneal-dialysis-related peritonitis. Int J Nephrol. 2012;2012: 483250.

3 Davenport A. Peritonitis remains the major clinical complication of peritoneal dialysis: The london, UK, peritonitis audit 2002-2003. Perit Dial Int. 2009 May-Jun;29(3):297-302.

4 Akoh JA. Peritoneal dialysis associated infections: An update on diagnosis and management. World J Nephrol. 2012 Aug;1(4):10622.

5 Krediet RT, Abrahams AC, de Fijter CW, Betjes MG, Boer WH, van Jaarsveld BC, et al. The truth on current peritoneal dialysis: state of the art. Neth J Med. 2017 Jun;75(5):179_ 89.
6 Davies SJ, Phillips L, Naish PF, Russell GI. Peritoneal glucose exposure and changes in membrane solute transport with time on peritoneal dialysis. J Am Soc Nephrol. 2001 May; 12(5):1046-51.

7 van Esch S, Struijk DG, Krediet RT. The natural time course of membrane alterations during peritoneal dialysis is partly altered by peritonitis. Perit Dial Int. 2016 Jul-Aug;36(4): $448-56$.

8 Krediet RT, Struijk DG. Peritoneal changes in patients on long-term peritoneal dialysis. Nat Rev Nephrol. 2013 Jul;9(7):419-29.

9 Nataatmadja M, Cho Y, Pascoe EM, Darssan D, Hawley CM, Johnson DW; balANZ Trial Investigators. Association between peritoneal glucose exposure and peritonitis in peritoneal dialysis patients: the balANZ trial. Perit Dial Int. 2017 Jul-Aug;37(4):407-13.

10 van Diepen AT, van Esch S, Struijk DG, Krediet RT. The association between glucose exposure and the risk of peritonitis in perito- neal dialysis patients. Perit Dial Int. 2016; 36(5):533-9.

11 Jiang N, Zhang Z, Fang W, Qian J, Mou S, Ni Z. High peritoneal glucose exposure is associated with increased incidence of relapsing and recurrent bacterial peritonitis in patients undergoing peritoneal dialysis. Blood Purif. 2015;40(1):72-8.

12 Han SH, Lee SC, Ahn SV, Lee JE, Kim DK, Lee $\mathrm{TH}$, et al. Reduced residual renal function is a risk of peritonitis in continuous ambulatory peritoneal dialysis patients. Nephrol Dial Transplant. 2007 Sep;22(9): 2653-8.

13 Liu YL, Huang CC, Kao MT. Residual renal function predicts outcome of fungal peritonitis in peritoneal dialysis patients. Perit Dial Int. 2006 May-Jun;26(3):407-9.

14 Marron B, Remon C, Perez-Fontan M, Quiros $\mathrm{P}$, Ortiz A. Benefits of preserving residual renal function in peritoneal dialysis. Kidney Int Suppl. 2008 Apr;(108):S42-51. 
15 Pérez Fontan M, Rodríguez-Carmona A, García-Naveiro R, Rosales M, Villaverde P, Valdés F. Peritonitis-related mortality in patients undergoing chronic peritoneal dialysis. Perit Dial Int. 2005 May-Jun;25(3):27484.

16 Davies SJ, Garcia Lopez E, Woodrow G, Donovan K, Plum J, Williams P, et al. Longitudinal relationships between fluid status, inflammation, urine volume and plasma metabolites of icodextrin in patients randomized to glucose or icodextrin for the long exchange. Nephrol Dial Transplant. 2008 Sep;23(9): 2982-8.

17 Li PK, Szeto CC, Piraino B, de Arteaga J, Fan $\mathrm{S}$, Figueiredo AE, et al. ISPD peritonitis rec- ommendations: 2016 update on prevention and treatment. Perit Dial Int. 2016 Sep;36(5): 481-508.

18 Davies SJ, Phillips L, Russell GI. Peritoneal solute transport predicts survival on CAPD independently of residual renal function. Nephrol Dial Transplant. 1998 Apr;13(4): 962-8.

19 Wu HY, Hung KY, Huang JW, Chen YM, Tsai TJ, Wu KD. Initial glucose load predicts technique survival in patients on chronic peritoneal dialysis. Am J Nephrol. 2008;28(5): 765-71.

20 Wu HY, Hung KY, Huang TM, Hu FC, Peng YS, Huang JW, et al. Safety issues of long-term glucose load in patients on peritoneal dialy- sis-a 7-year cohort study. PLoS One. 2012; 7(1):e30337.

21 Witowski J, Wisniewska J, Korybalska K, Bender TO, Breborowicz A, Gahl GM, et al. Prolonged exposure to glucose degradation products impairs viability and function of human peritoneal mesothelial cells. J Am Soc Nephrol. 2001 Nov;12(11):2434-41.

22 Mortier S, Lameire NH, De Vriese AS. The effects of peritoneal dialysis solutions on peritoneal host defense. Perit Dial Int. 2004 MarApr;24(2):123-38.

23 Struijk DG, Krediet RT, Koomen GC, Boeschoten EW, Hoek FJ, Arisz L. A prospective study of peritoneal transport in CAPD patients. Kidney Int. 1994 Jun;45(6):1739-44. 